\title{
Revista Colombiana de

\section{Modificación en el score de Framingham con la reducción de peso por cirugía bariátrica}

\section{Arturo Márquez ${ }^{\mathrm{a}, *}$, Marco-Fidel Chala $^{\mathrm{b}}$, Ricardo Bohórquez ${ }^{\mathrm{a}}$, Manuel Urina ${ }^{\mathrm{a}}$, Ángel García ${ }^{a}$ y Carlos Arias ${ }^{a}$}

\author{
a Servicio de Cardiología. Hospital Universitario San Ignacio, Bogotá, Colombia \\ b Clínica de obesidad, Hospital Universitario San Ignacio, Bogotá, Colombia
}

Recibido el 14 de diciembre de 2014; aceptado el 5 de agosto de 2015

Disponible en Internet el 28 de octubre de 2015

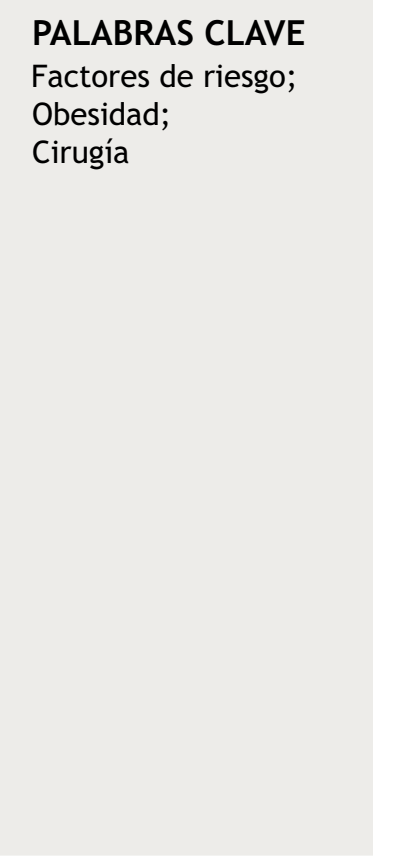

\begin{abstract}
Resumen
Objetivo: Evaluar el comportamiento de diferentes factores deriesgo y del score de Framingham con la disminución de peso obtenida a través de cirugía bariátrica al año postoperatorio, en una clínica de obesidad durante los años 2008 y 2011. Se evaluaron las modificaciones en los factores de riesgo y riesgo global con el score de Framingham al año postoperatorio, de una cohorte retrospectiva de 209 pacientes de la clínica de obesidad en un hospital universitario; los cambios se expresan como porcentajes de disminución respecto al valor prequirúrgico, en las variables nominales.

Resultados: El peso y el índice de masa corporal tuvieron una reducción del $48 \%$ y $38 \%$, respectivamente; el score de Framingham se redujo de 12,4 a 7,6 (35\%), es decir, pasó de riesgo intermedio a bajo (menor al 10\%). Así mismo, se observó disminución en los valores de hemoglobina glicosilada $(14,7 \%)$, colesterol total $(11,59 \%)$, colesterol-LDL $(7 \%)$ y triglicéridos $(31,4 \%)$, elevación del colesterol-HDL $(15,3 \%)$, suspensión del uso de insulina (18\%), disminución del $20 \%$ del valor de la glucosa y la HbA1c, a menos de 5,7 en un $23 \%$ de pacientes con glicemia alterada en ayunas, y finalmente, reducción de la presión arterial sistólica $(9,2 \%)$ y diastólica.

Conclusiones: La cirugía bariátrica es una herramienta válida en pacientes en quienes han fallado otros métodos clínicos para disminuir de peso e índice de masa corporal. Con ésta, además, se logra una disminución en el score de Framingham y se mejora el control de comorbilidades.

(c) 2015 Sociedad Colombiana de Cardiología y Cirugía Cardiovascular. Publicado por Elsevier España, S.L.U. Este es un artículo Open Access bajo la licencia CC BY-NC-ND (http:// creativecommons.org/licenses/by-nc-nd/4.0/).
\end{abstract}

\footnotetext{
* Autor para correspondencia.

Correo electrónico: amarquez235@hotmail.com (A. Márquez).
} 


\section{KEYWORDS}

Risk factors;

Obesity;

Surgery

\section{Framingham score modification with weight loss due to bariatric surgery}

\begin{abstract}
Objective: To assess the behaviour of different risk factors and Framingham score with weight loss, obtained with bariatric surgery one year after surgery, in an obesity clinic during 2008 and 2011.Modifications of risk factors and global risk of the Framingham score one year after surgery, of a retrospective cohort of 209 patients of the obesity clinic at a University hospital. Changes are expressed as percentages of the decrease with regard to the pre-surgical values, in nominal values.

Results: A $48 \%$ weight loss and $38 \%$ body mass index loss was observed, with a reduction of the Framingham score from 12.4 to $7.6(35 \%)$, moving from an intermediate risk to a low risk (below $10 \%$ ). A decrease of $14.7 \%$ in values of glycohemoglobin, $11.59 \%$ in total cholesterol, $7 \%$ of LDL cholesterol levels and $31.4 \%$ of triglycerides was observed, as well as a $15.3 \%$ raise in HDL cholesterol levels. $18 \%$ of patients with altered fasting glycaemia stopped taking insulin, glucose levels were reduced by $20 \%$ and A1c hemoglobin values were below 5.7 in $23 \%$ of the cases. Systolic pressure was reduced by $9.2 \%$, same as the diastolic blood pressure.

Conclusions: Surgery is a valid tool for patients were other clinical methods have failed, in order to lose weight and lower the body mass index, as well as reducing the Framingham score and improving the control of comorbidities.

(c) 2015 Sociedad Colombiana de Cardiología y Cirugía Cardiovascular. Published by Elsevier España, S.L.U. This is an open access article under the CC BY-NC-ND license (http:// creativecommons.org/licenses/by-nc-nd/4.0/).
\end{abstract}

\section{Introducción}

La obesidad es un problema serio de salud en el mundo; en Colombia, específicamente una de cada dos personas tiene sobrepeso u obesidad, entre ellas las más afectadas son las mujeres entre 18 y 29 años, con un 55,2\% y los hombres con el 45,6\%. En las áreas urbanas, uno de cada seis niños o adolescentes la padece y por departamentos los más afectados son San Andrés (65\%), Guaviare (62\%), Guainía (61\%), Vichada $(58,4 \%)$ y Caquetá $(58,8 \%)$.

Según la Encuesta nacional sobre situación nutricional 2010-ENSIN-, la obesidad mórbida se encuentra en $0,9 \%$, donde $0,3 \%$ corresponde a los hombres y $1,3 \%$ a las mujeres ${ }^{1}$. Así mismo, se asocia con incremento en la morbilidad y mortalidad por causas cardiovasculares ${ }^{2-4}$, y se estima que el costo para el tratamiento en Estados Unidos equivale al $9 \%$ del presupuesto en cuidado para la salud $^{5}$.

De otra parte, la asociación entre obesidad y enfermedad cardiovascular no se limita a los factores cardiovasculares tradicionales, pues existen mecanismos propios, como resistencia a la insulina y aumento en las concentraciones séricas de leptina, actividad simpática, tensión arterial, frecuencia cardiaca y actividad inflamatoria ${ }^{1-4}$.

Aproximadamente, un $50 \%$ de los obesos desarrollarán fibrilación auricular; por aumento en la vulnerabilidad eléctrica hay relación con QTc prolongado y con presencia de potenciales evocados tardíos, taquicardias y disminución de la variabilidad cardiaca, fenómeno que eleva el riesgo de muerte súbita ${ }^{4,5}$. Crece, así mismo, la prevalencia del síndrome de apnea del sueño, que a su vez incrementa el riesgo de eventos cardiovasculares y la incidencia de síndrome metabólico ${ }^{4,6}$ e hipertensión arterial de tres a seis veces más ${ }^{4,6}$.
El manejo de los cambios en el estilo de vida y las terapias farmacológicas arrojan resultados pobres; solo el $20 \%$ de los obesos adopta cambios en el estilo de vida. Los programas de actividad física con frecuencia son inefectivos. La utilidad de la terapia farmacológica también es limitada al comparar con placebo (eficacia a largo plazo de 2,9 a 4,3\%) ${ }^{5,6}$. El Instituto Nacional de Salud de Estados Unidos ha sugerido la cirugía como otra alternativa de tratamiento para pacientes con índice de masa corporal mayor a $40 \mathrm{~kg} / \mathrm{m}^{2}$ o mayor a $35 \mathrm{~kg} / \mathrm{m}^{2}$ asociada a presencia de comorbilidad ${ }^{7-9}$.

Estimar el riesgo cardiovascular es útil ya que permite identificar pacientes con alto riesgo de desarrollar cardiopatía isquémica al tiempo que sirve para guiar las estrategias de control del riesgo global ${ }^{10-12}$. Esta investigación permite conocer la epidemiología de pacientes con obesidad mórbida, a quienes se les realizó cirugía. En la actualidad, la cirugía se reserva para los casos de obesidad mórbida en quienes las estrategias de manejo médico han fallado y presentan un riesgo quirúrgico aceptable, mortalidad menor al $1 \%$. Dicha estrategia ha demostrado una mejoría significativa en la disminución de peso y en el control de comorbilidades $y$, en consecuencia, un aumento en la tasa de sobrevida ${ }^{13-15}$.

Con base en estudios hechos en otros países, se conoce como el tratamiento para la reducción del exceso de peso, minimiza la mortalidad global hasta en un $20 \%$, los valores de glicemia postprandial en un $50 \%$, las cifras de presión arterial sistólica y diastólica hasta en 10 y $20 \mathrm{~mm} \mathrm{Hg}$, respectivamente, el colesterol total en un $10 \%$, el colesterol LDL en $15 \%$ y los triglicéridos en un $30 \%$, en tanto que aumenta el colesterol HDL en $8 \%$, y a nivel hematológico disminuye la agregabilidad plaquetaria y mejora los procesos de fibrinólisis ${ }^{13,16,17}$. En Colombia no se conocen registros similares, y por tanto se consideró importante documentar estos hallazgos y compararlos con los de otros países. 
En este estudio se describe la prevalencia de factores de riesgo cardiovascular en pacientes colombianos con obesidad severa, a través de la evaluación del riesgo mediante el score de Framingham y su modificación al año de ser sometidos a cirugía bariátrica en un hospital universitario, durante el período 2008 a 2011.

Se evaluó el porcentaje de reducción de peso, índice de masa corporal, niveles de hemoglobina glicosilada en pacientes diabéticos, cambios en los niveles de glicemia en ayunas en pacientes con diagnóstico de prediabetes, efecto sobre el perfil lipídico a través de la medición de los niveles de colesterol total, colesterol-LDL, colesterol-HDL y triglicéridos, además del comportamiento de las cifras de tensión arterial sistólica y diastólica posteriores a la intervención.

Esta investigación de tipo observacional descriptivo, de cohorte retrospectiva, incluyó 316 pacientes operados en la clínica de obesidad de un hospital universitario en el período de 2008 a 2011, siguiendo los protocolos institucionales para ser sometidos a cirugía, como estrategia efectiva para reducir de peso. Se efectuó una descripción epidemiológica de los factores de riesgo cardiovascular y la predicción de riesgo de eventos cardiovasculares a diez años utilizando el score de Framingham en la primera fase y al año posterior a la intervención, y se evaluó el impacto sobre cada uno de los factores de riesgo y sobre el riesgo global de eventos coronarios a un año.

La población incluyó todo paciente mayor de 16 años, llevado a cirugía por obesidad mórbida y con valoración de los factores de riesgo mencionados antes de la intervención, además de aquellos que tuvieran datos de seguimiento clínico durante el primer año postoperatorio. Se tomaron las mediciones de las variables más cercanas al día de la cirugía y los análisis de las variables postquirúrgicas. Los datos demográficos y antropométricos de la población se extrajeron de la historia clínica e incluyeron peso, talla, índice de masa corporal, así como la presencia de hipertensión arterial, diabetes mellitus y dislipidemia.

Se excluyeron pacientes con seguimiento clínico extrainstitucional, aquellos con historia clínica incompleta o quienes no tenían descrita alguna de las variables a evaluar.

Las variables a medir incluyeron: género (masculino o femenino); edad en años, peso y altura (tomados de la base de recolección de datos); índice de masa corporal (calculado de acuerdo con la fórmula de peso en kilogramos dividido sobre la talla en $\mathrm{cm}$, elevada al cuadrado, tomando registros sobre valores promedios de las consultas) y perímetro abdominal en $\mathrm{cm}$ (tomado del promedio de mediciones, con datos posteriores a la intervención, que se compararon con los previos).

Las cifras de tensión arterial sistólica y diastólica en $\mathrm{mm}$ $\mathrm{Hg}$, se tomaron de los registros de la consulta de valoración prequirúrgica, con cambios en los valores absolutos y porcentuales de acuerdo con el estimativo de la mediana encontrada en las valoraciones previas y posteriores a la cirugía.

En cuanto a los valores de colesterol total, colesterolHDL y triglicéridos, cuando estos últimos no superaban los $400 \mathrm{mg} / \mathrm{dl}$ se estimaron indirectamente mediante la fórmula de Friedewald; cuando eran mayores a $400 \mathrm{mg} / \mathrm{dl}$ el colesterol LDL se calculó directamente.

Para la estratificación por score de Framingham se tomaron como variables, los criterios utilizados en el estudio original, usando la categorización de los factores de riesgo antes y después de la intervención. Las personas que fumaban regularmente en los últimos doce meses se clasificaron como fumadores.

La diabetes se consideró presente en los pacientes bajo tratamiento con insulina o agentes hipoglicemiantes orales o en quienes cumplían los criterios de la Asociación Americana de Diabetes para el diagnóstico de diabetes.

En lo concerniente al tipo de cirugía se consideraron cuatro posibilidades: banda gástrica, gastroplastia, bypass gástrico y derivación bilio-pancreática.

\section{Resultados}

Posterior al análisis estadístico de la base de datos de seguimiento de pacientes, se obtuvo un total de 316 participantes, de los cuales 209 cumplieron los criterios de inclusión. Se excluyeron aquellos a quienes se les realizó el procedimiento en fecha posterior a octubre de 2010 (107 casos) (fig. 1).

La edad promedio fue de 46,1 años (rango de edad entre los 16 años y los 72 años). El 87,05\% fueron mujeres, con promedio de índice de masa corporal de $44,2 \mathrm{~kg} / \mathrm{m}^{2}$ (DE 4,3) (rango de $35 \mathrm{a} 66 \mathrm{~kg} / \mathrm{m}^{2}$ ), y peso promedio de $111,79 \mathrm{~kg}$ (DE 16,17 ) (rango 170 a $76 \mathrm{~kg}$ ). La prevalencia de comorbilidades que aumentan el riesgo cardiovascular, identificó hipertensión arterial en el $48,5 \%(n=101)$, diabetes mellitus tipo 2 en el $29,1 \%(n=60)$ y dislipidemia en un $39,7 \%(n=83)$ de los casos.

Posterior al seguimiento de doce meses después de cirugía, la reducción del índice de masa corporal fue de 38\%; así, el índice de masa corporal disminuyó de 44,2 (DE 4,88) (rango 66 a 35) a $27,4 \mathrm{~kg} / \mathrm{m}^{2}$ (DE 2,71) (rango 40 a 21,7). Se observó una reducción del peso absoluto del 48\%; el peso promedio prequirúrgico fue $111,79 \mathrm{~kg}$ (DE 16,17) (rango 170 a $76 \mathrm{~kg}$ ) con descenso del peso a un promedio de $58,13 \mathrm{~kg}$ (DE 8,41) (rango 88,4 a 39,5 kg).

El control metabólico en pacientes con diabetes mellitus tipo 2 antes de la cirugía, definido por lograr valores de HbA1c menores de 6,5, correspondió al 47\% de los casos $(\mathrm{n}=32)$. El promedio del valor de HbA1c antes de la cirugía fue de $7,11 \%$ (DE 1,13) (rango 12,3-4,87) y al año postquirúrgico fue de 6,07 (DE 0,73) (rango de 10,9 a 4,5), cifra que representa un descenso de $1,04 \%$ de $\mathrm{HbA} 1 \mathrm{c}$, equivalente al $14,6 \%$. Adicionalmente, en un $18 \%$ de los pacientes que requerían tratamiento con insulina como parte del manejo

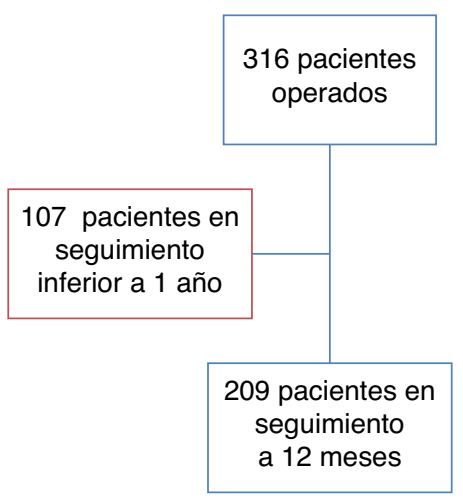

Figura 1 Población intervenida con cirugía bariátrica por obesidad mórbida. 
de la diabetes, se logró suspender el medicamento al punto de corte.

Los pacientes con glicemia alterada en ayunas, mostraron una disminución del promedio de glicemia de ayuno de $20,7 \%$ $(114,35 \mathrm{~g} / \mathrm{dl}$ a $90,57 \mathrm{~g} / \mathrm{dl})$, así como de la HbA1c a valores normales menores al $5,7 \%$ en el $23 \%$ de los pacientes de este grupo.

Se halló modificación de las cifras de tensión arterial en el $59,3 \%$ de los pacientes, con disminución de 10,7 $( \pm 2,2 \mathrm{~mm}$ $\mathrm{Hg}$ ) en la tensión arterial sistólica y 6,36 ( $\pm 1,02 \mathrm{~mm} \mathrm{Hg})$ en la diastólica.

Se documentaron cambios en el perfil lipídico, con descenso de niveles promedio de colesterol total de 207 a $183 \mathrm{mg} / \mathrm{dl}$, equivalentes a $11,59 \%$, aumento de $\mathrm{HDL}$ de $42,86 \mathrm{mg} / \mathrm{dl}$ a $50,61 \mathrm{mg} / \mathrm{dl}$ correspondientes al 15,31\%; descenso del LDL medido de 114,37 a 106,15 mg/dl equivalente al $7,01 \%$ y disminución de triglicéridos de $178 \mathrm{mg} / \mathrm{dl}$ a $122 \mathrm{mg} / \mathrm{dl}$ correspondiente al 31,46\% al año.

El score de Framingham prequirúrgico promedio en la población intervenida, era de $12,41 \%$, catalogado como moderado, y al año postoperatorio disminuyó significativamente a 7,6\%, representando así el 35\% de reducción relativa de riesgo de enfermedad coronaria para los siguientes diez años.

\section{Discusión}

La cirugía bariátrica constituye una herramienta útil para lograr una disminución de peso de manera significativa y sostenida en el tiempo, en pacientes con diagnóstico de obesidad mórbida asociada a comorbilidades que aumentan de manera exponencial el riesgo de enfermedad cardiovascular. La literatura mundial describe que la disminución de peso alcanza hasta el $50 \%$, principalmente en pacientes sometidos a cirugía de derivación (bypass gástrico). De esta manera, la asociación de reducción de peso en esta proporción, induce mejor control de las cifras de tensión arterial, glicemia y perfil lipídico, que finalmente se reflejarán en la disminución de los marcadores de riesgo para desarrollar enfermedad cardiovascular, constituyendo una medida de prevención primaria y secundaria.

Bien se sabe que el beneficio de mayor impacto se ha descrito en el control de la diabetes, con descenso en los valores de $\mathrm{HbA} 1 \mathrm{c}$ que incluso llegan a la normalidad, al igual que la disminución en el requerimiento de insulina o terapias farmacológicas euglicemiantes orales. Adicionalmente, en este estudio se documentó un impacto importante en el grupo de prediabéticos, con disminución y control de la glicemia de ayuno alterada luego de la intervención. El impacto sobre la hipertensión arterial, fue mayor y significativo para este grupo de seguimiento en valores de presión arterial sistólica. En lo que atañe a los resultados en el análisis de cambios en el perfil lipídico, llama la atención el control, en un porcentaje considerable de pacientes, sobre los triglicéridos y el colesterol LDL y HDL respectivamente.

Estos resultados son reproducibles a la luz de la evidencia actual, ya que se halló un descenso del $35 \%$ en el score de Framingham para enfermedad coronaria a diez años. Cabe resaltar que el grupo de pacientes intervenidos en este estudio, se encuentra dentro de la categoría de riesgo intermedio $(12 \%)$, por lo que se infiere que la toma de la decisión podría realizarse de manera precoz e intervenir a la misma población con un menor puntaje de riesgo y menor lesión de órgano diana. También es notorio el límite de edad, mayor en este registro con respecto a la mayoría de análisis mundiales, con puntos de corte de edad avanzada mayor de 65 años (5\% de la muestra), a diferencia de los grupos descritos en el resto del mundo, en los que el promedio máximo de edad fue inferior a 55 años.

\section{Conclusión}

Esta intervención muestra claros beneficios en la prevención primaria y secundaria de la obesidad severa, mostrando una relación costo-beneficio positiva para enfermedad coronaria y obesidad severa.

\section{Responsabilidades éticas}

Protección de personas y animales. Los autores declaran que para esta investigación no se han realizado experimentos en seres humanos ni en animales.

Confidencialidad de los datos. Los autores declaran que han seguido los protocolos de su centro de trabajo sobre la publicación de datos de pacientes.

Derecho a la privacidad y consentimiento informado. Los autores declaran que en este artículo no aparecen datos de pacientes.

\section{Conflicto de intereses}

Los autores declaran no tener ningún conflicto de intereses.

\section{Bibliografía}

1. Encuesta Nacional de la Situación Nutricional. 2010. ENSIP; Ministerio de Salud, Instituto Nacional de Salud, Asociación Colombiana para el Avance de la Ciencia, Instituto Colombiano de Bienestar Familiar, DANE. El Tiempo.com.co, 2013, Cifras Obesidad en Colombia.

2. Poirier P, Giles TD, Bray GA, Hong Y, Stern JS, Pi-Sunyer FX, et al. American Heart Association; Obesity Committee of the Council on Nutrition, Physical Activity, and Metabolism. Obesity and cardiovascular disease: pathophysiology, evaluation, and effect of weight loss: an update of the 1997 American Heart Association Scientific Statement on Obesity and Heart Disease from the Obesity Committee of the Council on Nutrition, Physical Activity, and Metabolism. Circulation. 2006;113:898-918.

3. Sturm R. Increases in clinically severe obesity in the United States, 1986-2000. Arch Intern Med. 2003;163:2146-8.

4. Poirier P, Giles TD, Bray GA, Hong Y, Stern JS, Pi-Sunyer FX, Eckel $\mathrm{RH}$. American Heart Association; Obesity Committee of the Council on Nutrition, Physical Activity, and Metabolism. Obesity and cardiovascular disease: pathophysiology, evaluation, and effect of weight loss: an update of the 1997 American Heart Association Scientific Statement on Obesity and Heart Disease from the Obesity Committee of the Council on Nutrition, Physical Activity, and Metabolism. Circulation. 2006;113:898-918.

5. Poirier P, Cornier MA, Mazzone T, Stiles S, Cummings S, Klein $S$, et al. Bariatric surgery and cardiovascular risk factors: a scientific statement from the American Heart Association. Circulation. 2011;123:1683-701. 
6. Cooney MT, Dudina A, D’Agostino R, Graham IM. Cardiovascular risk estimation systems in primary prevention. Circulation. 2011;123:1683-701.

7. Oishansky SJ, Passaro Dento J, Hershow RC, Layden J, Carnes BA, Brady J, et al. A potencial decline in life in the United States in the $21^{\text {st }}$ century. N Engl J Med. 2005;352:1138-45.

8. Kenchaiah S, Evans JC, Levy D, Wilson PWF, Benjamin EJ, Larson MG, et al. Obesity and the risk of hearth failure. N Engl J Med. 2002;347(5):305-13.

9. Calle EE, Thun MJ, Petrelli JM, Rodriguez C, Heath Jr. CW. Bodymass index and mortality in a prospective cohort of U.S. adults. N Engl J Med. 1999;(15):1097-105.

10. Anderson KM, Wilson PW, Odell PM, Kannel WB. An updated coronary risk profile: a statement for health professionals. Circulation. 1991;83:356-62.

11. Brotons C. Mejoremos la predicción del riesgo coronario en España. Rev Esp Cardiol. 2003;56:225-77.

12. Assmann G, Cullen P, Schulte H. Simple scoring scheme for calculating the risk of acute coronary events based on the 10-year follow-up of the Prospective Cardiovascular Munster (PROCAM) Study. Circulation. 2002;105:310-59.
13. Poirier P, Alpert MA, Fleisher LA, Thompsom PD, Sugerman HJ, Burke LE, et al. Impact of bariatric surgery on cardiac structure, function and clinical manifestations in morbid obesity. Expert Rev Cardiovasc Ther. 2004;2(2):195-201.

14. Guidelines for reporting results in bariatric surgery: Standards Committee, American Society for Bariatric Surgery. Obes Surg. 1997; 7:521-2.

15. Mechanick JI, Kushner RF, Sugerman HJ, Gonzalez-Campoy JM, Collazo-Clavell ML, Guven S, et al. American Association of Clinical Endocrinologists, The Obesity Society, and American Society for Metabolic \& Bariatric Surgery Medical guidelines for clinical practice for the perioperative nutritional, metabolic, and nonsurgical support of the bariatric surgery patient. Endocr Pract. 2008;14 suppl1:1-83.

16. Batsis JA, Sarr MG, Collazo-Clavell ML, Thomas RJ, RomeroCorral A, Somers VK, et al. Cardiovascular risk after bariatric surgery for obesity. Am J Cardiol. 2008;102:930-7.

17. Batsis JA, Romero-Corral A, Collazo-Clavell ML, Sarr MG, Somers VK, Lopez-Jimenez F. Effect of bariatric surgery on the metabolic syndrome: a population-based, long-term controlled study. Mayo Clin Proc. 2008;83:897-907. 